\title{
Surface hydrogenation in diffuse and translucent interstellar clouds
}

\author{
P. T. O'neill, S. Viti, and D. A. Williams \\ University College London, Gower Street, London WC1E 6BT, UK \\ Received 15 March 2001 / Accepted 28 March 2002

\begin{abstract}
We investigate the effects of grain-surface hydrogenation reactions on gas phase chemical models of diffuse and translucent clouds in the interstellar medium. Models in which gas phase species freeze out on to dust without release do not match observed column densities well. Expanding on previous work we extend our models to include the release of $\mathrm{C}, \mathrm{N}, \mathrm{O}, \mathrm{S}$ and $\mathrm{CO}$ following hydrogenation on the grain surface. The results show that such mechanisms do improve the ability of chemical models to reproduce observed abundances, not only through the release of otherwise frozen-out species but also because of the additional hydrogenating reactions. For example, the predicted column densities of $\mathrm{NH}$ in diffuse clouds and $\mathrm{H}_{2} \mathrm{~S}$ and $\mathrm{NH}_{3}$ in translucent clouds match observations better in models with grain-surface hydrogenation.
\end{abstract}

Key words. astrochemistry - molecular processes - ISM: abundances - ISM: molecules

\section{Introduction}

Diffuse and translucent clouds remain something of an enigma in modern astronomy. Although the longeststudied of interstellar clouds, their role in the evolution of matter in space is unclear. Their relatively simple chemistry has led them to be identified as test-beds of chemical networks. This may be inappropriate, as they present difficulties (for example, the pathological case of $\mathrm{CH}^{+}$; and the high observed abundance of $\mathrm{CO}$ ) that have not currently been resolved. It seems evident that both the dynamics and the detailed pressure-density structure may be affecting the chemistry. In addition, uncertainties in some gas phase reactions remain, and the contribution of grain-surface reactions - indicated by both $\mathrm{H}_{2}$ and $\mathrm{NH}$ - cannot yet be regarded as definitive, owing to limited experimental and theoretical support. However, this last point is being addressed in several studies (Vidali et al. 1999; Pirronello et al. 1999; Williams et al. 1999).

It is encouraging, therefore, that there has been a resurgence of interest in observations of diffuse and translucent clouds, and the list of identified molecular species has been significantly extended. Comprehensive lists of identified interstellar molecules are given in van Dishoeck (1998), Turner (2000) and Lucas \& Liszt (1997). Recently, two new important species have been observed in the interstellar medium: $\mathrm{CH}_{3}$ (Feuchtgruber et al. 2000) and $\mathrm{C}_{3}$ (Maier et al. 2001; Haffner \& Meyer 1995). Most of these new discoveries cannot be adequately accounted for on the basis of simple chemical models, and

Send offprint requests to: S. Viti,

e-mail: sv@star.ucl.ac.uk either more complex cloud structures (Nguyen et al. 2001) or additional chemical routes have been proposed.

In an earlier paper (Viti et al. 2000) we explored the potential contribution of grain surface reactions to hydrocarbon formation in diffuse and translucent clouds as a supplement to the well-established gas phase networks. The gas phase network alone appears insufficient as a model of these clouds because it is thought that when a molecule in the gas meets a dust grain it will stick to the surface (Williams 1998). Some energetic mechanism must be involved if the molecule is to return to the gas phase, and a chemical change may well occur (Jones \& Williams 1984). In the previous paper two models were explored: in the first (Model A) it was simply assumed that all $\mathrm{C}^{+}$ ions arriving at grain surfaces were incorporated into hydrogenated amorphous carbon (HAC), and in the second (Model B) the $\mathrm{C}^{+}$ion was assumed to be returned to the gas as $\mathrm{CH}_{4}$, thereby feeding the hydrocarbon chemical network. A hybrid of these models was also discussed. The results showed that Model A could not account for the chemical richness observed, but that Model B, and the hybrid model $(\mathrm{AB})$, could provide an adequate match to the observations of hydrocarbons in diffuse and translucent clouds. The abundances of several as yet undetected species were also predicted.

One of the more significant problems in diffuse cloud chemistry has been the failure of models to account adequately for the abundant molecule CO (Wagenblast \& Williams 1993). Gas phase formation routes to CO include $\mathrm{C}$ and $\mathrm{C}^{+}$with $\mathrm{OH}, \mathrm{O}$ with $\mathrm{CH}$, and $\mathrm{HCO}^{+}$with $\mathrm{e}^{-}$. The formation route through the $\mathrm{CH}$ and $\mathrm{O}$ gas phase reaction is enhanced by the adoption of $\mathrm{CH}_{4}$ injection from 
grains (Viti et al. 2000) but even in this case there still appears to be a shortfall in the computed CO abundance. Wagenblast \& Williams (1996) suggested that an additional contribution from dust-formed $\mathrm{OH}$ and $\mathrm{H}_{2} \mathrm{O}$ reacting with $\mathrm{C}^{+}$might resolve the difficulty. Hence it seems reasonable to extend the chemistry of Viti et al. (2000) to include the surface hydrogenation of other species on dust grains, and to explore the contribution of the product molecules to the gas phase network of reactions.

We have, therefore, included hydrogenation of $\mathrm{O}, \mathrm{N}$, $\mathrm{S}^{+}$, CO to various products which then enter the gas phase network. These gas + grain chemistry models are explored for a variety of environments, ranging from rather low number density, low extinction clouds, to moderate density, moderate extinction clouds.

In Sect. 2 we describe the details of the models, including the adopted hydrogenation efficiency, largely as in Viti et al. (2000). The effects of the hydrogenation reactions are described in Sect. 3. The main results are given in the form of column densities integrated through the clouds, as in Viti et al. (2000), in Sect. 4, where we compare our models with some of the available observations. In Sect. 5 we briefly present our conclusions.

\section{The models}

The basic chemical model which we use is the UMIST rate file (Millar et al. 1997) with the slight modifications described in Viti et al. (2000). The initial elemental abundances by number relative to hydrogen were taken to be $7.0 \times 10^{-2}, 1.1 \times 10^{-4}, 6.0 \times 10^{-5}, 4.6 \times 10^{-4}, 1.3 \times 10^{-5}$, $3.0 \times 10^{-9}, 2.0 \times 10^{-7}$ and $7.0 \times 10^{-9}$ for helium, carbon, nitrogen, oxygen, sulphur, magnesium, sodium, and silicon respectively. These values are taken from Snow \& Witt (1996) as in the previous paper, though more recent estimates differ. For example, Savage \& Sembach (1996) give abundance for $\mathrm{Mg}$ and $\mathrm{Si}$ more than two orders of magnitude different, although those changes in particular would not significantly affect the results that we shall present. The adopted value of the cosmic ray ionization rate $\zeta$, is $1.3 \times 10^{-17} \mathrm{~s}^{-1}$. For static slab model clouds with densities of each of 100, 300 and $1000 \mathrm{H}$-nuclei $\mathrm{cm}^{-3}$ we run time-dependent chemical multipoint calculations to find the gas phase abundances for evolutionary times up to $10^{7}$ years, over a range of visual extinction from 0 to 4 mag. CO self-shielding is accounted for using codes developed by Wagenblast (1992). The gas is assumed to be evenly mixed with dust grains to which molecules will stick on collision: a process known as "freeze out". The efficiency of freeze out for a given molecule depends on the size and charge of the grains; assuming, as is usual, that any species contacting a grain will stick with probability $\approx 1$. In our models we use a traditional grain model. All grains are assumed to have an excess charge of $1 \mathrm{e}^{-}$.

There are alternative grain models which expand the traditional grain size distribution to include large numbers of very small grains. In such a case the small grains will dominate surface processes because they present most of the available surface area. The effects of the grain surface processes we examine in this paper could be significantly changed in such models: firstly, because the smaller grains will tend to have a significantly different charge distribution (Weingartner \& Draine 1999), altering not only the freeze-out rates but also possibly affecting the ionization balance between atoms and their corresponding ions (Lepp et al. 1988); and secondly, because the distribution of frozen-out species is less likely to be constant across all grains, it may not be possible to model surface processes in a classical manner.

We compare results from eleven different chemistries: Models A and B are as mentioned in Sect. 1; Model A has no recovery of species from grains, and in Model B all positive carbon ions that meet the grain surfaces are reinjected into the gas phase as $\mathrm{CH}_{4}$ molecules. The other chemical models that we used add extra hydrogenation reactions to Model B as described below. In all cases, the products of these surface reactions are assumed to be returned promptly to the gas phase.

Model C extends Model B by including the hydrogenation of oxygen and nitrogen: $\mathrm{O}$ forms $\mathrm{H}_{2} \mathrm{O}$ on collision with a dust grain, and $\mathrm{N}$ forms $\mathrm{NH}_{3}$.

Model D adds to Model $\mathrm{C}$ the hydrogenation of sulphur: $\mathrm{S}^{+}$is hydrogenated to $\mathrm{H}_{2} \mathrm{~S}$ on grains.

We also tested three models to examine consequences of the hydrogenation of CO. In Models E1 to E3 hydrogenation of $\mathrm{CO}$ into different species is added to the chemistry of Model $\mathrm{C}$, that is, with $\mathrm{C}^{+}, \mathrm{O}$ and $\mathrm{N}$ hydrogenation. In Model E1, CO forms $\mathrm{H}_{2} \mathrm{CO}$ on grains, and in Model E2 the product is $\mathrm{CH}_{3} \mathrm{OH}$. Model E3 is a hybrid of $\mathrm{E} 1$ and $\mathrm{E} 2$ in which $\mathrm{H}_{2} \mathrm{CO}$ and $\mathrm{CH}_{3} \mathrm{OH}$ are formed equally. Models F1 to F3 are the same as E1 to E3 but with the hydrogenation of $\mathrm{S}^{+}$added as in Model D.

Finally, in addition to the above we have included results from a model using a chemistry identical to that of Model A (without any hydrogenation on grains) but in which species do not freeze out on to dust grains. We call this Model $\mathrm{Z}$ and it is provided as a reference to show the situation where dust grains do not interact chemically with the gas phase. The hydrogenation reactions added in each model are summarized in Table 1.

\section{Effects of the hydrogenation reactions}

The changes in computed abundances with the addition of new grain-surface reactions are essentially as expected. Release of molecules from grains to the gas phase will necessarily cause an increase in abundance in all models relative to model A for all species based on the elements released. This is simply because an element that was being removed from the gas phase in Model A is still present in other models. If there is an ejection mechanism for one hydrogenated species (as in Model B), then it seems logical to expect ejection of some kind for other hydrogenated species; the real question is the form of the ejection reactions and their relative efficiencies. Unfortunately, not enough is yet known about activity on the grains to set 
Table 1. Summary of hydrogenation reactions on grain surfaces included in each chemical model.

\begin{tabular}{cl}
\hline \hline Chemical model & Surface hydrogenation reactions \\
\hline A & None (freeze-out of all species) \\
B & $\mathrm{C}^{+} \downarrow \longrightarrow \mathrm{CH}_{4} \uparrow$ \\
C & As Model B but with $\mathrm{O} \downarrow \longrightarrow \mathrm{H}_{2} \mathrm{O} \uparrow$, and $\mathrm{N} \downarrow \longrightarrow \mathrm{NH}_{3} \uparrow$ also \\
D & As C, with $\mathrm{S}^{+} \downarrow \longrightarrow \mathrm{H}_{2} \mathrm{~S} \uparrow$ \\
E1 & As C, with $\mathrm{CO} \downarrow \longrightarrow \mathrm{H}_{2} \mathrm{CO} \uparrow$ \\
E2 & As C, with $\mathrm{CO} \downarrow \longrightarrow \mathrm{CH}_{3} \mathrm{OH} \uparrow$ \\
E3 & As C, with $50 \% \mathrm{CO} \downarrow \longrightarrow \mathrm{H}_{2} \mathrm{CO} \uparrow, 50 \% \mathrm{CO} \downarrow \longrightarrow \mathrm{CH}_{3} \mathrm{OH} \uparrow$ \\
F1 & As D, with $\mathrm{CO} \downarrow \longrightarrow \mathrm{H}_{2} \mathrm{CO} \uparrow$ \\
F2 & As D, with $\mathrm{CO} \downarrow \longrightarrow \mathrm{CH}_{3} \mathrm{OH} \uparrow$ \\
F3 & As D, with $50 \% \mathrm{CO} \downarrow \longrightarrow \mathrm{H}_{2} \mathrm{CO} \uparrow, 50 \% \mathrm{CO} \downarrow \longrightarrow \mathrm{CH}_{3} \mathrm{OH} \uparrow$ \\
Z & Purely gas-phase chemistry (as A) with no grain interaction \\
\hline
\end{tabular}

accurate a priori rates; and current observational data are probably not extensive enough to make detailed studies of such complex systems. Hence, we introduce ejection reactions for only a few species at a time, each with $100 \%$ efficiency: this efficiency may be unrealistic, but at this stage we are only interested in studying the qualitative effects of surface grain chemistry on the evolution of diffuse and translucent clouds in order to answer the following question: would the abundances of hydrogenated species vary enough to produce a detectable difference? By introducing a $100 \%$ efficiency of ejection, we are, of course, allowing for the largest possible effect.

We have calculated the abundances for visual extinctions from 0 to 4 mags for clouds of density 100, 300 and $1000 \mathrm{~cm}^{-3}$. This enables us to consider the chemistry in several régimes including diffuse clouds with density $\leq 300 \mathrm{~cm}^{-3}$ and $A_{V} \sim 1$ mags, and translucent clouds with higher density and $A_{V} \sim 3$ mags. Tables 2-4 summarize our results; they contain column densities for the clouds after a time of $10^{7}$ years-there is no steady state for models with freeze-out. In Tables 2 and 3 Col. 9 shows the observed column densities of $\zeta$ Per (in Table 2) and $\zeta$ Oph (in Table 3) taken from the literature; for some species, however, these were not available in which cases we have compared our models with the column densities derived along different lines of sight (not presented in the tables) and discussed these comparisons in the text (in particular Sects. 4.1.1, 4.1.3 and 4.1.5). Even when available, we have not included error bars in listing the observed values, as comparisons between observations and our model results should only be done in qualitative terms. Note that the column densities of Models A and B may differ from the ones listed in Viti et al. (2000) because we have incorporated a better treatment of $\mathrm{CO}$ self-shielding and a more accurate estimation of the integrated column densities.

Here we review in qualitative terms the consequences of introducing surface hydrogenation. The effects of adding hydrogenation reactions for $\mathrm{C}^{+}$were discussed in the previous paper (Viti et al. 2000); in Model C where we have added hydrogenation for oxygen and nitrogen there is an equivalent set of changes for species containing these elements. Of particular interest are the $\mathrm{H}_{2} \mathrm{O}$ and $\mathrm{CO}$ abundances which are related because $\mathrm{H}_{2} \mathrm{O}$ and $\mathrm{OH}$ are thought to be part of a major formation route for $\mathrm{CO}$. The introduction of surface hydrogenation for $\mathrm{C}^{+}$(Model B) increases the amount of $\mathrm{CH}_{2}$ present, which reacts with oxygen to produce $\mathrm{CO}$. The column density of $\mathrm{CO}$ is thereby greatly increased (compared to Model A), but the column densities of $\mathrm{H}_{2} \mathrm{O}$ and $\mathrm{OH}$ in Model $\mathrm{B}$ fall compared to Model A: this is a consequence of the increase in carbonbearing species in the gas. With the addition of surface hydrogenation of oxygen directly into $\mathrm{H}_{2} \mathrm{O}$ (Model $\mathrm{C}$ ) the former $\mathrm{C}: \mathrm{O}$ ratio is maintained and the column densities of $\mathrm{H}_{2} \mathrm{O}$ and $\mathrm{OH}$ recover to approximately the levels of Model A.

The addition of surface hydrogenation of nitrogen to $\mathrm{NH}_{3}$ in Model $\mathrm{C}$ affects directly the abundance of $\mathrm{NH}_{3}$, increasing it well above the level of Models A and B.

With the inclusion of sulphur hydrogenation to $\mathrm{H}_{2} \mathrm{~S}$ in Model D, the column densities of $\mathrm{S}$ and $\mathrm{S}^{+}$are increased for all cloud densities. For the diffuse clouds these are the only significant effects. However, at the translucent cloud density of $1000 \mathrm{~cm}^{-3}$ several species, including CS, $\mathrm{SO}$ and $\mathrm{H}_{2} \mathrm{~S}$, have column densities increased by several orders of magnitude.

The differences between Models E1, E2 and E3 are negligible. The only species whose column density changes significantly between them is $\mathrm{CH}_{3} \mathrm{OH}$. It seems that $\mathrm{H}_{2} \mathrm{CO}$ can be adequately produced from $\mathrm{CH}_{3} \mathrm{OH}$ in the gas phase so there is no real need to consider these models (or F1F3) separately. In the tables of results therefore we have only included Models E3 and F3 which both produce $\mathrm{CH}_{3} \mathrm{OH}$ and $\mathrm{H}_{2} \mathrm{CO}$ in equal amounts from CO. Probably the most interesting thing to observe with these models is that they do not decrease the column density of $\mathrm{CO}$; indeed, in the translucent cloud case it is increased even further beyond that of Model C, probably as a consequence of the increase of the carbon budget in the gas due to 
Table 2. Results of chemical models for 1 mag of visual extinction for clouds with number density of $100 \mathrm{~cm}^{-3}$ after $10^{7}$ years. The computed column densities (in $\mathrm{cm}^{-2}$ ) of each species from seven models are compared to the observed column densities for $\zeta$ Per taken from the literature, when available. (Note, numbers in the form $a(b)$ above represent $a \times 10^{b}$.)

\begin{tabular}{lllllllll}
\hline \hline Species & Model & & & & & & \multicolumn{2}{l}{ Observation } \\
& $\mathrm{A}$ & $\mathrm{B}$ & $\mathrm{C}$ & $\mathrm{D}$ & $\mathrm{E} 3$ & $\mathrm{~F} 3$ & $\mathrm{Z}$ & \multicolumn{1}{c}{$\zeta$ Per } \\
\hline $\mathrm{H}$ & $1.7(20)$ & $1.7(20)$ & $1.7(20)$ & $1.7(20)$ & $1.7(20)$ & $1.7(20)$ & $1.7(20)$ & $8.9(20)-1.4(21)^{a}$ \\
$\mathrm{H}_{2}$ & $7.2(20)$ & $7.2(20)$ & $7.2(20)$ & $7.2(20)$ & $7.2(20)$ & $7.2(20)$ & $7.2(20)$ & $3.2(20)-7.1(20)^{a}$ \\
$\mathrm{~S}$ & $6.4(11)$ & $5.0(12)$ & $5.0(12)$ & $6.0(13)$ & $5.0(12)$ & $6.0(13)$ & $5.9(13)$ & $1.8(13)^{b}$ \\
$\mathrm{~S}^{+}$ & $2.0(15)$ & $2.0(15)$ & $2.0(15)$ & $2.1(16)$ & $2.0(15)$ & $2.1(16)$ & $2.1(16)$ & $1.6(16)^{c}$ \\
$\mathrm{CS}$ & $8.0(7)$ & $5.3(9)$ & $5.3(9)$ & $6.7(10)$ & $5.3(9)$ & $6.7(10)$ & $2.4(10)$ & $-^{*}$ \\
$\mathrm{SO}$ & $1.5(7)$ & $1.5(7)$ & $1.9(7)$ & $1.7(9)$ & $1.9(7)$ & $1.7(9)$ & $2.0(8)$ & $-^{*}$ \\
$\mathrm{CO}$ & $8.5(11)$ & $1.5(13)$ & $3.1(13)$ & $3.0(13)$ & $3.1(13)$ & $3.0(13)$ & $1.1(13)$ & $6.1(14)^{b}$ \\
$\mathrm{C}_{2} \mathrm{H}$ & $1.4(8)$ & $8.3(11)$ & $8.3(11)$ & $8.0(11)$ & $8.3(11)$ & $8.0(11)$ & $1.0(11)$ & $-^{*}$ \\
$\mathrm{C}_{3} \mathrm{H}_{2}$ & $5.2(4)$ & $2.0(9)$ & $2.0(9)$ & $1.7(9)$ & $2.0(9)$ & $1.7(9)$ & $1.8(8)$ & $-^{*}$ \\
$\mathrm{C}_{2} \mathrm{H}_{2}$ & $3.2(6)$ & $3.2(10)$ & $3.2(10)$ & $3.2(10)$ & $3.2(10)$ & $3.2(10)$ & $2.9(8)$ & - \\
$\mathrm{CH}_{3}$ & $4.2(9)$ & $1.4(12)$ & $1.4(12)$ & $1.4(12)$ & $1.4(12)$ & $1.4(12)$ & $1.9(10)$ & - \\
$\mathrm{NH}^{*}$ & $1.4(10)$ & $9.2(9)$ & $3.1(11)$ & $3.1(11)$ & $3.1(11)$ & $3.1(11)$ & $1.1(10)$ & $1(12)^{d}$ \\
$\mathrm{CN}$ & $3.1(8)$ & $7.7(9)$ & $6.8(10)$ & $6.7(10)$ & $6.8(10)$ & $6.7(10)$ & $5.2(9)$ & $3.0(12)^{e}$ \\
$\mathrm{OH}$ & $1.0(13)$ & $9.9(11)$ & $3.9(12)$ & $3.8(12)$ & $3.9(12)$ & $3.8(12)$ & $1.1(12)$ & $4.0(13)^{e}$ \\
$\mathrm{H}_{3}^{+}$ & $3.8(13)$ & $4.5(12)$ & $4.5(12)$ & $4.1(12)$ & $4.5(12)$ & $4.1(12)$ & $4.0(12)$ & $-^{*}$ \\
$\mathrm{C}_{3}$ & $2.5(4)$ & $2.9(9)$ & $2.9(9)$ & $2.7(9)$ & $2.9(9)$ & $2.7(9)$ & $2.9(8)$ & $-^{*}$ \\
\hline
\end{tabular}

${ }^{a}$ Savage et al. (1977), ${ }^{b}$ Snow (1977), ${ }^{c}$ Snow et al. (1987), ${ }^{d}$ Meyer \& Roth (1991), e Felenbok \& Roueff (1996), * some species for which $\zeta$ Per observations are not available have however been observed for other clouds; we discuss this in the text.

the ejection and dissociation of $\mathrm{CH}_{3} \mathrm{OH}$ and $\mathrm{H}_{2} \mathrm{CO}$. Also in the translucent cloud models the amount of $\mathrm{CO}_{2}$ is increased and we can see an increase in the column densities of $\mathrm{H}_{2} \mathrm{CO}$ and $\mathrm{CH}_{3} \mathrm{OH}$ themselves. The hydrogenation of $\mathrm{CO}$ makes no appreciable difference in the diffuse cloud regime.

Model Z, without freeze-out, naturally differs most from Model A in which all species suffer freeze-out with no ejection. One of the main effects of the postulated hydrogenation and ejection mechanism is simply avoiding depletion of elements from the gas phase. This restores to models other than A more of the characteristics of Model Z. However, it is thought that ions and molecules which meet the surface of a dust grain will stick to it, making Model Z seem unphysical. The differences between this model and others due to hydrogenation rather than the simple release of frozen species (notably $\mathrm{CH}_{3}$ and $\mathrm{NH}$ at low densities and $\mathrm{H}_{2} \mathrm{~S}$ and $\mathrm{NH}_{3}$ at $1000 \mathrm{~cm}^{-3}$ ) may offer some support for ejection from grains as a result of chemical reactions on the surface.

\section{Comparisons with observations}

Observationally, diffuse and translucent clouds have been extensively studied. We have taken into consideration as many relevant studies as possible, although for a more comprehensive summary we refer the reader to several recent reviews: Turner (2000), who summarizes the results of a large observational study of translucent clouds; a review by van Dishoeck (1998) where recent results on translucent and diffuse clouds are presented; and Lucas \& Liszt (1997) who present comprehensive observations of diffuse clouds. The relation of our results to observations is discussed below.

\subsection{Diffuse clouds}

We choose to compare results of selected species from models of number densities of $100 \mathrm{~cm}^{-3}$ (cf. Table 2, close to the $\zeta$ Per number density) and $300 \mathrm{~cm}^{-3}$ (cf. Table 3 , close to the $\zeta$ Oph number density) with observations of diffuse clouds. Many of the species in the tables have been selected because they have been observed towards the lines of sight of $\zeta$ Per, $\zeta$ Oph and other diffuse clouds.

\subsubsection{Sulphur-bearing species}

Atomic and ionized sulphur have been observed along the line of sight to $\zeta$ Per (Snow 1977; Snow et al. 1987) and $\xi$ Per (Smith et al. 1991). The column density of neutral sulphur is estimated to be approximately $1.8 \times 10^{13} \mathrm{~cm}^{-2}$ by Snow (1977) and slightly lower $\left(7.24-9.55 \times 10^{12} \mathrm{~cm}^{-2}\right)$ by Smith et al. (1991). More significant is the ratio of $N(\mathrm{C}) / N(\mathrm{~S})$, given by the latter authors as $\sim 80$, significantly larger than obtained from previous work (GomezGonzalez \& Lequeux 1975). As the number densities of $\zeta$ Per and $\xi$ Per are close to $100 \mathrm{~cm}^{-3}$ (Wagenblast et al. 1993; Smith et al. 1991), we compare these observations of 
Table 3. Results of chemical models for $1 \mathrm{mag}$ of visual extinction for clouds with density of $300 \mathrm{~cm}^{-3}$ after $10^{7}$ years. The computed column densities (in $\mathrm{cm}^{-2}$ ) of each species from seven models are compared to the observed column density for $\zeta$ Oph taken from the literature, when available.

\begin{tabular}{lllllllll}
\hline \hline Species & Model & & & & & & \multicolumn{2}{c}{ Observation } \\
& $\mathrm{A}$ & $\mathrm{B}$ & $\mathrm{C}$ & $\mathrm{D}$ & $\mathrm{E} 3$ & $\mathrm{~F} 3$ & $\mathrm{Z}$ & $\zeta$ Oph \\
\hline $\mathrm{H}$ & $5.5(19)$ & $6.0(19)$ & $6.1(19)$ & $6.0(19)$ & $6.1(19)$ & $6.0(19)$ & $5.9(19)$ & $5.2(20)^{a}$ \\
$\mathrm{H}_{2}$ & $7.7(20)$ & $7.7(20)$ & $7.7(20)$ & $7.7(20)$ & $7.7(20)$ & $7.7(20)$ & $7.7(20)$ & $4.5(20)^{b}$ \\
$\mathrm{~S}$ & $6.8(9)$ & $1.0(11)$ & $1.0(11)$ & $1.3(14)$ & $1.0(11)$ & $1.3(14)$ & $1.3(14)$ & $8.5(13)^{c}$ \\
$\mathrm{~S}^{+}$ & $1.7(13)$ & $1.8(13)$ & $1.8(13)$ & $2.1(16)$ & $1.8(13)$ & $2.1(16)$ & $2.1(16)$ & $1.2(16)^{c}$ \\
$\mathrm{CS}$ & $3.1(3)$ & $5.1(8)$ & $5.0(8)$ & $6.1(11)$ & $5.0(8)$ & $6.1(11)$ & $2.0(11)$ & $7(11)^{-5}(12)^{d}$ \\
$\mathrm{SO}$ & $7.1(5)$ & $7.3(5)$ & $1.3(6)$ & $1.2(10)$ & $1.3(6)$ & $1.2(10)$ & $1.4(9)$ & $-{ }^{*}$ \\
$\mathrm{CO}$ & $1.9(9)$ & $6.0(13)$ & $2.2(14)$ & $2.1(14)$ & $2.2(14)$ & $2.1(14)$ & $6.9(13)$ & $1.72(15)^{e}$ \\
$\mathrm{C}_{2} \mathrm{H}$ & $4.6(2)$ & $5.0(12)$ & $4.9(12)$ & $4.6(12)$ & $4.9(12)$ & $4.6(12)$ & $5.9(11)$ & $-{ }^{*}$ \\
$\mathrm{C}_{3} \mathrm{H}_{2}$ & $3.5(-4)$ & $3.3(10)$ & $3.2(10)$ & $2.6(10)$ & $3.2(10)$ & $2.6(10)$ & $2.7(9)$ & $-{ }^{*}$ \\
$\mathrm{C}_{2} \mathrm{H}_{2}$ & $1.8(1)$ & $2.3(11)$ & $2.3(11)$ & $2.2(11)$ & $2.3(11)$ & $2.2(11)$ & $2.1(9)$ & - \\
$\mathrm{CH}_{3}$ & $1.6(7)$ & $2.9(12)$ & $2.8(12)$ & $2.8(12)$ & $2.8(12)$ & $2.8(12)$ & $4.6(10)$ & - \\
$\mathrm{NH}$ & $9.7(9)$ & $5.3(9)$ & $6.5(11)$ & $6.5(11)$ & $6.5(11)$ & $6.5(11)$ & $8.8(9)$ & $8.8(11)^{f}$ \\
$\mathrm{CN}$ & $7.4(5)$ & $3.9(10)$ & $4.2(11)$ & $4.0(11)$ & $4.2(11)$ & $4.0(11)$ & $2.8(10)$ & $3(12)^{g}$ \\
$\mathrm{OH}$ & $1.7(13)$ & $6.8(11)$ & $6.9(12)$ & $6.7(12)$ & $6.9(12)$ & $6.7(12)$ & $1.0(12)$ & $4.8(13)^{h}$ \\
$\mathrm{H}_{3}^{+}$ & $3.3(13)$ & $1.8(12)$ & $1.8(12)$ & $1.6(12)$ & $1.8(12)$ & $1.6(12)$ & $1.5(12)$ & $-{ }^{*}$ \\
$\mathrm{C}_{3}$ & $1.5(-4)$ & $4.9(10)$ & $4.8(10)$ & $4.2(10)$ & $4.8(10)$ & $4.3(10)$ & $4.5(9)$ & $1.6(12)^{a}$ \\
\hline
\end{tabular}

${ }^{a}$ Maier et al. (2001), ${ }^{b}$ Bohlin et al. (1978), ${ }^{c}$ Morton (1975), ${ }^{d}$ Drdla et al. (1989), ${ }^{e}$ Lambert et al. (1994), ${ }^{f}$ Crawford \& Williams (1997), ${ }^{g}$ Lambert et al. (1990), ${ }^{h}$ Chaffee \& Lutz (1977), ${ }^{*}$ some species for which $\zeta$ Oph observations are not available have however been observed for other clouds; we discuss this in the text.

atomic sulphur with values quoted in Table 2. Models B and $\mathrm{C}$ are close to the lower limit of Smith et al. while Model $\mathrm{D}$ is four times larger than the value quoted by Snow et al. However, if we compare the $N(\mathrm{C}) / N(\mathrm{~S})$ ratio, Models D and Z both give a ratio of 30 , lower than the observed value (Smith et al. 1991), though close to the older value $\sim 32$ found by Gomez-Gonzalez \& Lequeux (1975). By contrast, in models where ionized sulphur is frozen out the ratio is $\sim 340$, much larger than observed, suggesting that sulphur is not being retained on the grains in these clouds.

Ionized sulphur is observed to have a column density of $\approx 1.6 \times 10^{16} \mathrm{~cm}^{-2}$ (Snow 1977; Snow et al. 1987), three orders of magnitude larger than neutral sulphur. This ratio is roughly consistent with our Models B, C and D. As expected, the $\mathrm{S}^{+} / \mathrm{S}$ ratio does not vary among models, since grain hydrogenation and ejection of ionized sulphur would increase the total sulphur budget in the gas phase. However, the observed ionized sulphur column density is well matched by Model D, and also by Model Z.

Drdla et al. (1989) have searched the CS $J=2-1$ emission line in 10 diffuse molecular clouds and successfully detected it in four of the clouds, including $\zeta \mathrm{Oph}$. They derive the total CS column densities from one line by means of statistical equilibrium calculations and they found that the CS column density varied from $\sim 2 \times 10^{12}$ to $3 \times 10^{13} \mathrm{~cm}^{-2}$. Lucas \& Liszt (1997) also detected molecular absorption of CS and SO, among other species, in front of extragalactic millimetre wavelength radio sources and find column densities for both species between $10^{12}$ and $10^{13} \mathrm{~cm}^{-2}$. Their cloud density is, however, larger than the one adopted by our models, probably few hundred $\mathrm{cm}^{-3}$ on average (Lucas \& Liszt 1997).

From Tables 2 and 3 one can see that CS is only formed in significant amounts when sulphur freeze-out and hydrogenation occur. In Model D for densities of at least $300 \mathrm{~cm}^{-3}$, CS reaches the lower limit of the observed abundances. In general, we find that our models underestimate the abundance of CS in the diffuse medium although Model D is very close to the observed lower limit. A slightly higher density than that used for Table 3 would improve the agreement.

We conclude from the analysis of sulphur-bearing species that models with grain surface reactions involving the hydrogenation of sulphur are favoured over those models with freeze-out. However, in the diffuse cloud case, there is little to distinguish these models from a model without freeze-out.

\subsubsection{Carbon monoxide}

Viti et al. (2000) found that surface hydrogenation of $\mathrm{C}^{+}$ indirectly yields an increase in CO by more than $30 \%$ but that the computed column density was more than one order of magnitude less than that observed for $\zeta$ Oph (but note that this line of sight has an unusually high $\mathrm{CO}$ column density, and may not be typical).

Table 3 shows a clear increase in column density from Model B to Model C, where oxygen and nitrogen 
Table 4. Results of chemical models for $3 \mathrm{mag}$ of visual extinction for clouds with density of $1000 \mathrm{~cm}^{-3}$ after $10^{7}$ years. The computed column densities (in $\mathrm{cm}^{-2}$ ) of each species from seven models are compared to column densities for translucent clouds taken from the literature. With the exception of the column densities from van Dishoeck et al. (1991) the observational values presented are determined from fractional abundances averaged over a variety of translucent clouds, and assuming a gas column density around $3 \times 10^{21}$.

\begin{tabular}{lllllllll}
\hline \hline Species & Model & & & & & & Observation \\
& $\mathrm{A}$ & $\mathrm{B}$ & $\mathrm{C}$ & $\mathrm{D}$ & $\mathrm{E} 3$ & $\mathrm{~F} 3$ & $\mathrm{Z}$ & \\
\hline $\mathrm{H}$ & $7.7(18)$ & $1.8(19)$ & $1.9(19)$ & $1.8(19)$ & $1.9(19)$ & $1.8(19)$ & $1.6(19)$ & - \\
$\mathrm{H}_{2}$ & $2.4(21)$ & $2.4(21)$ & $2.4(21)$ & $2.4(21)$ & $2.4(21)$ & $2.4(21)$ & $2.4(21)$ & $\sim 3(22)$ \\
$\mathrm{CS}$ & $1.4(5)$ & $4.9(5)$ & $2.1(7)$ & $1.8(13)$ & $2.7(7)$ & $2.2(13)$ & $2.7(13)$ & $3.3(13)^{a}$ \\
$\mathrm{SO}$ & $2.7(5)$ & $5.8(4)$ & $3.1(8)$ & $1.6(13)$ & $1.8(8)$ & $1.7(13)$ & $2.0(12)$ & $9.6(13)^{a}$ \\
$\mathrm{H}_{2} \mathrm{~S}$ & $1.0(3)$ & $2.2(2)$ & $1.6(5)$ & $3.2(13)$ & $1.1(5)$ & $3.2(13)$ & $4.7(9)$ & $7.5(13)^{a}$ \\
$\mathrm{NH}_{3}$ & $5.3(11)$ & $2.2(11)$ & $2.3(13)$ & $1.9(13)$ & $2.1(13)$ & $1.8(13)$ & $9.8(9)$ & $3-6(13)^{a}$, \\
$\mathrm{N}_{2} \mathrm{H}^{+}$ & $5.9(10)$ & $2.8(10)$ & $4.1(11)$ & $7.2(9)$ & $2.3(11)$ & $5.3(9)$ & $8.7(8)$ & $3(12)^{a}$ \\
$\mathrm{HCN}$ & $7.3(10)$ & $1.0(11)$ & $3.5(11)$ & $3.3(11)$ & $5.1(11)$ & $5.2(11)$ & $5.3(10)$ & $1.1(14)^{a}$ \\
$\mathrm{HNC}$ & $1.0(11)$ & $9.7(10)$ & $1.5(12)$ & $1.4(12)$ & $1.5(12)$ & $1.5(12)$ & $3.5(10)$ & $7.5(12)^{a}$ \\
$\mathrm{CH}{ }_{3} \mathrm{OH}$ & $4.4(9)$ & $2.7(9)$ & $1.1(10)$ & $1.3(9)$ & $3.2(13)$ & $2.5(13)$ & $1.6(8)$ & $1.8(13)^{c}$ \\
$\mathrm{CO}$ & $8.3(15)$ & $3.4(16)$ & $8.0(16)$ & $6.6(16)$ & $2.9(17)$ & $2.5(17)$ & $2.2(17)$ & $1(16)^{c}-1(17)^{d}$ \\
$\mathrm{H}_{3}^{+}$ & $1.6(14)$ & $3.0(13)$ & $3.4(13)$ & $1.1(13)$ & $2.8(13)$ & $9.9(12)$ & $5.7(12)$ & - \\
$\mathrm{C}_{2} \mathrm{H}$ & $4.1(11)$ & $9.1(13)$ & $2.8(13)$ & $2.1(13)$ & $3.0(13)$ & $2.4(13)$ & $6.2(12)$ & $1.98(14)^{e}$ \\
$\mathrm{C}_{3} \mathrm{H}_{2}$ & $9.3(9)$ & $4.4(12)$ & $6.0(11)$ & $3.5(11)$ & $6.3(11)$ & $4.3(11)$ & $1.1(11)$ & $3(12)^{f}$ \\
$\mathrm{CH}_{3}$ & $1.6(12)$ & $1.4(13)$ & $6.6(12)$ & $5.8(12)$ & $1.1(13)$ & $9.6(12)$ & $3.4(11)$ & - \\
$\mathrm{C}_{3}$ & $4.1(9)$ & $3.3(12)$ & $6.4(11)$ & $4.6(11)$ & $7.4(11)$ & $5.8(11)$ & $1.8(11)$ & - \\
\hline
\end{tabular}

${ }^{a}$ Turner (2000), ${ }^{b}$ Turner (1995a), ${ }^{c}$ Turner (1998), ${ }^{d}$ van Dishoeck et al. (1991), ${ }^{e}$ Turner et al. (1999), ${ }^{f}$ Cox et al. (1988).

are hydrogenated on grains. Although CO is still underabundant by at least half an order of magnitude with respect to the CO observed in that line of sight (see Table 1 in Viti et al. 2000), the results suggest that if freezeout is added to the gas-phase chemistry model used here, then some release from the grains is necessary for model abundances to approach observations. A somewhat higher diffuse cloud density would improve the match between Model $\mathrm{C}$ values and that of $\zeta \mathrm{Oph}$.

\subsubsection{Hydrocarbons}

A full discussion of hydrocarbon chemistry was given in Viti et al. (2000). However, due to the extension of our surface chemistry to hydrogenation of oxygen and $\mathrm{CO}$, which may affect the abundances of hydrocarbons, and recent detections by Lucas \& Liszt (2000) and Feuchtgruber et al. (2000) we briefly discuss the hydrocarbons $\mathrm{C}_{3} \mathrm{H}_{2}, \mathrm{C}_{2} \mathrm{H}$ and $\mathrm{CH}_{3}$.

Lucas \& Liszt (2000) have detected absorption lines of $\mathrm{C}_{2} \mathrm{H}$ and $\mathrm{C}_{3} \mathrm{H}_{2}$ along many lines of sight. They find column densities of, respectively, $\sim 7 \times 10^{12} \mathrm{~cm}^{-2}$ and $\sim 4.8 \times 10^{12} \mathrm{~cm}^{-2}$. From Table 3 it is obvious that purely gas phase low temperature models cannot account for the detection of these species. Viti et al. (2000) concluded that surface hydrogenation and ejection of hydrogenated species from the grains may be necessary to account for hydrocarbon abundances. In fact, Model B fails to meet the Lucas \& Liszt column densities by less than one order of magnitude in the case of $\mathrm{C}_{2} \mathrm{H}$ and about one order of magnitude for $\mathrm{C}_{3} \mathrm{H}_{2}$. Lucas \& Liszt (2000) imply a gas density of $\sim 200 \mathrm{~cm}^{-3}$, similar to ours; however, at a gas density of $1000 \mathrm{~cm}^{-3}$, our column density matches extremely well the abundances measured by Lucas \& Liszt (2000) (Table 4). Model A, where no hydrogenation and ejection occurs is clearly inadequate, regardless of the cloud density. Note that van Dishoeck \& Black (1986) have succeeded in reproducing the lower limit of the observed column densities of $\mathrm{C}_{2} \mathrm{H}$ and $\mathrm{C}_{3} \mathrm{H}$ (and other hydrocarbons in general) without invoking grain surface reactions, however, their models do not include depletion of gas phase species on to the grain, a process which is now believed to occur in some degree (Williams 1998).

Viti et al. (2000) proposed to test the hypothesis that $\mathrm{CH}_{4}$ is made and released on grains by detecting its products formed in the gas phase; $\mathrm{CH}_{3}$ is indeed the most direct product but direct comparison with this species (rather than with one of its products, $\mathrm{H}_{2} \mathrm{CO}$ ) had not yet been considered because at the time of the analysis no observations of $\mathrm{CH}_{3}$ were available. Recently, Feuchtgruber et al. (2000) detected $\mathrm{CH}_{3}$ towards the Galactic Centre and deduced a column density of $(8.0 \pm 2.4) \times 10^{14} \mathrm{~cm}^{-2}$. Our models fail to match the observed value by at least one order of magnitude (see Tables 2-4). However, we note that (i) the gas density along the line of sight observed by Feuchtgruber et al. (2000) is believed to be $>1000 \mathrm{~cm}^{-3}$ : comparisons of Tables 2-4 indicate that the $\mathrm{CH}_{3}$ column density increases proportionally to the gas density; (ii) if no hydrogenation and ejection is included (i.e. Model A), the basic ion-molecule gas-phase chemistry networks cannot account for the formation of any of the $\mathrm{CH}_{3}$ detected. Even without any freeze-out (Model Z) the 
predicted column density is nearly two orders of magnitude below those of models with hydrogenation of carbon.

\subsubsection{Nitrogen-bearing species}

Among the nitrogen-bearing species, we discuss $\mathrm{NH}$ and CN. NH was first detected by Meyer \& Roth (1991) towards $\zeta$ Per and HD 27778 and found to have a column density of $\sim 10^{12} \mathrm{~cm}^{-2}$. More recently, Crawford \& Williams (1997) estimated its column density towards $\zeta$ Oph from much higher resolution data to be $\sim 8.8 \times 10^{11} \mathrm{~cm}^{-2}$. Lambert et al. (1990) reported a column density of CN towards $\zeta$ Oph of $\sim 3 \times 10^{12} \mathrm{~cm}^{-2}$. $\mathrm{NH}$ is very well reproduced by models where nitrogen hydrogenation and ejection occur. CN, on the other hand, is under-produced by almost one order of magnitude, although, again, in the models where no hydrogenation occurs CN cannot be formed in the gas phase in detectable quantities. Note that previous models, such as the ones by Wagenblast \& Williams (1993), produce much higher CN abundance than we do in Model A: this is because their models do not include depletion of the gas phase on to the grains. Our equivalent model without depletion (Model Z), though better than Model A, is still worse than models with hydrogenation at predicting $\mathrm{NH}$ and $\mathrm{CN}$ abundances in diffuse clouds.

\subsection{5. $\mathrm{OH}$ and $\mathrm{H}_{3}^{+}$}

Chaffee \& Lutz (1977) detected OH towards $\zeta$ Oph and reported a column density of $\sim 4.8 \times 10^{13} \mathrm{~cm}^{-2}$. Geballe et al. (1999) detected $\mathrm{H}_{3}^{+}$for the first time in diffuse interstellar clouds (towards Cygnus OB2) and found a high column density of $\sim 3.8 \times 10^{14} \mathrm{~cm}^{-2}$. Computed abundances for both species are greater (and closer to observations) in models where freeze-out without ejection of hydrogenated species occurs, i.e. in Model A, although in the case of $\mathrm{OH}$ from Table 2 (where the density is supposed to be representative of $\zeta \mathrm{Oph}$ ), there is less than half an order of magnitude difference between Model A (very close to observations) and Model $\mathrm{C}$ or E3. The fact that $\mathrm{OH}$ and $\mathrm{H}_{3}^{+}$are more abundant when freeze-out occurs without hydrogenation and ejection is not surprising as ions are extremely reactive and they are readily removed when hydrogenated species such as water are abundant in the gas. In fact, we note that in Model A more than $50 \%$ of the $\mathrm{OH}$ is produced via dissociative recombination of $\mathrm{H}_{3} \mathrm{O}^{+}$while in the other Models this route only accounts for $\sim 10 \%$ of the $\mathrm{OH}$ present. This may be the cause for the difference between a model where only freeze-out occurs and one where freeze-out plus hydrogenation and ejection occurs. $\mathrm{H}_{3} \mathrm{O}^{+}$is easily destroyed by species such as $\mathrm{HCN}$ and HNC: column densities of these species are more than two orders of magnitude larger in models where hydrogenation occurs than in Model A.

Compared to the observed value, $\mathrm{H}_{3}^{+}$is underabundant in all our models. Note however that the (Geballe et al. 1999) detection of $\mathrm{H}_{3}^{+}$was towards
Cygnus OB2, at a visual extinction of $\sim 10$ mag: comparing Tables 3 and 4 , we note that $\mathrm{H}_{3}^{+}$increases by over one order of magnitude when $A_{V}$ increases from 1 to 3 mags: this should not be due to a density increase because comparison of Tables 2 and 3 (same $A_{V}$ but different density) shows that $\mathrm{H}_{3}^{+}$decreases with density (in fact, Geballe et al. 1999 note that the $\mathrm{H}_{3}^{+}$column density is not proportional to the column densities of other species). We infer that a model where the density is kept to $\sim 300 \mathrm{~cm}^{-3}$ and $A_{V}$ increased to 10 mags should match the observed $\mathrm{H}_{3}^{+}$column density, even for models where hydrogenation and ejection occurs.

Another factor which may explain the discrepancy between the computed $\mathrm{OH}$ and $\mathrm{H}_{3}^{+}$abundances and the observed values is the cosmic ray ionization rate, $\zeta$. The abundances of both these species are directly related to $\zeta$ through their dependence on $\mathrm{H}_{2}^{+}$which is formed by ionization of $\mathrm{H}_{2}$ by cosmic rays. $\mathrm{H}_{2}^{+}$reacts with $\mathrm{H}_{2}$ to form $\mathrm{H}_{3}^{+}$directly, and $\mathrm{OH}$ is formed by dissociative recombination of $\mathrm{H}_{3} \mathrm{O}^{+}$which is the product of $\mathrm{H}_{3}^{+}$and $\mathrm{H}_{2} \mathrm{O}$. In our models $\zeta$ is taken to be $1.3 \times 10^{-17} \mathrm{~s}^{-1}$ but in diffuse clouds a value of $\zeta$ between $10^{-17} \mathrm{~s}^{-1}$ and $10^{-16} \mathrm{~s}^{-1}$ is plausible (van Dishoeck 1998). If $\zeta$ were increased by half an order of magnitude in our models the computed abundances could be brought close to those observed.

\subsection{6. $\mathrm{C}_{3}$}

Haffner \& Meyer (1995) reported a tentative detection of $\mathrm{C}_{3}$ towards HD 147889. More recently Maier et al. (2001) observed $\mathrm{C}_{3}$ in diffuse interstellar clouds along the lines of sight towards three stars, including $\zeta \mathrm{Oph}$, and inferred a column density for $\mathrm{C}_{3}$ of $1.0-2.0 \times 10^{12} \mathrm{~cm}^{-2} \cdot \mathrm{C}_{3}$ is one of only two bare carbon species detected in diffuse clouds and it is a potential carrier for some of the Diffuse Interstellar Bands (Maier et al. 2001). Our diffuse clouds models (see Tables 2 and 3) do not reproduce the high column density observed. However, hydrogenation of $\mathrm{C}^{+}$ (Models B-F) does offer some improvement over models with no freeze-out or no ejection.

\subsection{Translucent clouds}

Many species have been observed in translucent clouds (Turner 2000; van Dishoeck 1998). Here, we select some observational results with which we may compare our model results. Apart from $\mathrm{CH}_{3} \mathrm{OH}$, we shall not discuss any other hydrocarbons as they were the subject of our earlier paper (Viti et al. 2000).

\subsubsection{Sulphur-bearing species}

At number densities of the order of $1000 \mathrm{~cm}^{-3}$, the column density of many sulphur-bearing species increases when ionized sulphur is hydrogenated on the grains (Models D and F). The most significant feature of the results is that these species should not be detectable if sulphur freezes out as in Models A to C. But sulphur-bearing species have been observed extensively in translucent clouds. For 
example, Turner (1995b, 1996a,b) conducted a survey in a standard sample of 11 Cirrus cores and 27 ClementBarvainis translucent objects. Table 6 in Turner (1996a) summarizes his sulphur studies by listing the ratios of sulphur-bearing species abundances in all the observed sources. He divides his objects between Cirrus and Clemens-Barvainis $(\mathrm{CB})$ cores and finds $\mathrm{CS} / \mathrm{SO}=$ 0.056-0.196, and $\mathrm{H}_{2} \mathrm{~S} / \mathrm{SO}=0.21-0.982$ for the Cirrus cores; whereas $\mathrm{CS} / \mathrm{SO}=0.047-0.478$, and $\mathrm{H}_{2} \mathrm{~S} / \mathrm{SO}=$ 0.263-3.4 for the $\mathrm{CB}$ objects (note that the observed column densities listed in Table 4 for CS, SO and $\mathrm{H}_{2} \mathrm{~S}$ are taken from Table 1 in Turner 2000). The $\mathrm{H}_{2} \mathrm{~S} / \mathrm{CS}$ ratios vary between 1.10 and 34.5. From Model D we have: $\mathrm{CS} / \mathrm{SO}=0.3, \mathrm{H}_{2} \mathrm{~S} / \mathrm{SO}=0.65$ and $\mathrm{H}_{2} \mathrm{~S} / \mathrm{CS}=2.1$. Our CS/SO ratio is within the observational values for the $\mathrm{CB}$ objects and $\mathrm{H}_{2} \mathrm{~S} / \mathrm{SO}$ and $\mathrm{H}_{2} \mathrm{~S} / \mathrm{CS}$ are between the (somewhat loose) limits given by the observations. Our conclusion for sulphur is that since these species are widely detected in translucent clouds, surface hydrogenation and ejection are occurring. Note that grain surface reactions have been invoked by Turner (1996a) in order to explain observed sulphur bearing species (e.g. $\mathrm{H}_{2} \mathrm{~S}$ ). The improved match of Model D to observations of SO and especially $\mathrm{H}_{2} \mathrm{~S}$ over Model $\mathrm{Z}$ suggests that hydrogenation and ejection may be preferable to ejection without surface reaction.

\subsubsection{Nitrogen-bearing species}

For translucent clouds, we find that all nitrogen-bearing species in Model C increase by 1-2 orders of magnitude compared to Model B. For example, the fractional abundance of $\mathrm{NH}_{3}$ at $A_{V}=3$ mags is $7.65 \times 10^{-10}$ for Model B and $1 \times 10^{-8}$ for Model $\mathrm{C}$, with a slight decrease in Model D. Turner (1995a) has found a fractional abundance of $\sim 1-2 \times 10^{-8}$ which, assuming $A_{V}=3$ mags, corresponds to a column density $\sim 3-6 \times 10^{13} \mathrm{~cm}^{-2}$. Model $\mathrm{C}$ results are in agreement with this measurement. Turner (2000) reports a fractional abundance for $\mathrm{N}_{2} \mathrm{H}^{+}$of $\sim 10^{-9}$ which, assuming $A_{V}=3$ mags, corresponds to a column density of $\sim 3 \times 10^{12} \mathrm{~cm}^{-2}$. The model which predicts the closest match to the observations is Model $\mathrm{C}$ : in this model gas phase $\mathrm{NH}_{3}$, and therefore most of the nitrogenbearing species in the gas, are enhanced with respect to Models A and B; however Model D (where gas phase $\mathrm{NH}_{3}$ is enhanced as well) predicts a much lower $\mathrm{N}_{2} \mathrm{H}^{+}$column density than even Model A and B (where N freezes and remains on the grains); this is probably due to the fact that in Model D gas phase $\mathrm{H}_{2} \mathrm{~S}$, and eventually neutral sulphur are enhanced and the reaction with sulphur is the main route of destruction for $\mathrm{N}_{2} \mathrm{H}^{+}$.

The HCN column density is reported to be at least $\sim 6 \times 10^{11} \mathrm{~cm}^{-2}$ by Liszt \& Lucas (1994). Models E3 and F3 are in very good agreement with this lower limit. These models have a rich chemistry due to the hydrogenation of nitrogen, carbon, oxygen and $\mathrm{CO}$ on grains. A puzzling result is, however, the variation of $\mathrm{HCN} / \mathrm{HNC}$ ratio among models: in models where hydrogenation and ejection of nitrogen occur, the HNC column density increases, while that of $\mathrm{HCN}$ stays roughly the same as in Models $\mathrm{A}$ and $\mathrm{B}$, leading to a decrease in the $\mathrm{HCN} / \mathrm{HNC}$ ratio (making it much less than unity) for Models $\mathrm{C}-$ F3; this result is not supported by observations. Indeed, Turner et al. (1997) find the ratio HCN/HNC to be "significantly above unity in translucent clouds". In fact, the decrease of this ratio in our models may be misleading: both HCN and HNC fractional abundances do indeed increase when nitrogen is hydrogenated and released from grains but while HCN is efficiently destroyed, HNC remains constant. The observational result of Turner et al. (1997) therefore suggests that the UMIST95 rate file, which we have used, may not contain all the possible destruction routes for HNC. Indeed the new UMIST99 database (Le Teuff et al. 2000) does add several new reactions for HNC.

\subsubsection{Methanol}

In the previous paper we found that none of our models could produce methanol in the quantities that are observed, and we noted Turner's suggestion (Turner 1998) that methanol may form on grains. In addition there is some experimental work examining the conversion of $\mathrm{CO}$ to $\mathrm{CH}_{3} \mathrm{OH}$ (Hudson \& Moore 1999).

Models E2, E3, F2 and F3 include, at either 50 or $100 \%$ efficiency, a surface reaction producing methanol from CO sticking on grains. There is, of course, an increase in methanol column density over other models. In fact, even Models E3 and F3, where only $50 \%$ of hydrogenated $\mathrm{CO}$ produces methanol, give a column density which is an order of magnitude higher than observed. Thus if $\mathrm{CO}$ is indeed hydrogenated on grains the efficiency required would need to be only a few percent in order to reproduce observations.

\section{Conclusions}

We began from the premise that if $\mathrm{C}^{+}$can be hydrogenated and ejected from the surface of grains, as suggested in a previous paper (Viti et al. 2000), then a similar process may well exist for other elements such as nitrogen, oxygen and sulphur. In a hydrogen-rich environment it seems likely that such elements, when frozen out, will become hydrogenated (Jones \& Williams 1984). As mentioned above, models such as our Model A which include depletion onto grains but no ejection fall well short of reproducing observed column densities for several molecules.

In this paper we have explored the possible consequences of introducing dust-based hydrogenation mechanisms into our gas-phase chemical models. We have taken the view that atoms, atomic ions and $\mathrm{CO}$ may either be retained at the surface, or be hydrogenated and ejected into the gas phase. We have also briefly compared with results obtained assuming freeze-out does not occur (equivalent to immediate ejection without any surface reactions).

The results for both low density diffuse and translucent clouds show that hydrogenation and ejection from 
the surface of grains does improve the ability of models to reproduce the observed values for some species. In particular, the re-release of oxygen into the gas phase does help bring the predicted CO column density of our models closer to the observed values in diffuse clouds. Models B-F do not predict $\mathrm{H}_{3}^{+}$high column density observed in diffuse clouds, although we believe that this is simply due to the low visual extinction adopted in the models.

For translucent clouds, differences among models appear to be even more significant. Observations of sulphurbearing species seem to suggest that this element must avoid depletion in some way, and ejection following a hydrogenation reaction matches observation better than simply avoiding freeze-out. Our models including hydrogenation of $\mathrm{CO}$ suggest that this may be a good formation route for methanol and a suitable choice of efficiency could easily match observation. These models (and indeed all others) still maintain the CO column density within observed limits. One contradictory result is the inversion of the $\mathrm{HCN} / \mathrm{HNC}$ ratio when nitrogen is hydrogenated. This may have arisen because the UMIST95 rate file does not include all possible destruction routes for HNC.

Overall, the outlook for these kinds of mechanisms is favourable. Given correct knowledge of rates and products they may help to explain some observed trends or features. After submission of this paper, new observations of diffuse and translucent clouds were published (Lucas \& Liszt 2002; McCall et al. 2002; Roueff et al. 2002), underlining the renewed interest in the chemistry and physics of these regions. Theoretical and experimental studies of surface processes and interactions are clearly needed in order to understand more fully the chemistry of diffuse and translucent clouds.

Acknowledgements. PTO acknowledges receipt of a PPARC studentship and DAW also wishes to acknowledge receipt of financial support from PPARC. We would also like to thank the referee, Professor John Black, for his very helpful comments that have greatly improved an earlier version of this paper.

\section{References}

Bohlin, R. C., Savage, B. D., \& Drake, J. F. 1978, ApJ, 224, 132

Chaffee, F. H. J., \& Lutz, B. L. 1977, ApJ, 213, 394

Cox, P., Gusten, R., \& Henkel, C. 1988, A\&A, 206, 108

Crawford, I. A., \& Williams, D. A. 1997, MNRAS, 291, L53

Drdla, K., Knapp, G. R., \& van Dishoeck, E. F. 1989, ApJ, 345,815

Felenbok, P., \& Roueff, E. 1996, ApJ, 465

Feuchtgruber, H., Helmich, F. P., van Dishoeck, E. F., \& Wrigth, C. M. 2000, ApJ, 535, 111L

Geballe, T. R., McCall, B. J., Hinkle, K. H., \& T, O. 1999, ApJ, 510, 251

Gomez-Gonzalez, J., \& Lequeux, J. 1975, A\&A, 38, 29

Haffner, L. M., \& Meyer, D. M. 1995, ApJ, 453

Hudson, R. L., \& Moore, M. H. 1999, Icarus, 140, 451

Jones, A. P., \& Williams, D. A. 1984, MNRAS, 209, 955

Lambert, D. L., Sheffer, Y., \& Crane, P. 1990, ApJ, 359, 19L

Lambert, D. L., Sheffer, Y., Gilliland, R. L., \& Federman, S. R. 1994, ApJ, 420, 756L
Le Teuff, Y. H., Millar, T. J., \& Markwick, A. J. 2000, A\&AS, 146,147

Lepp, S., Dalgarno, A., \& van Dishoeck, E. F. 1988, ApJ, 329, 418

Liszt, H., \& Lucas, R. 1994, A\&A, 282, 5L

Lucas, R., \& Liszt, H. 1997, in Molecules in Astrophysics: probes and processes, ed. E. van Dishoeck, 421

Lucas, R., \& Liszt, H. S. 2000, A\&A, 358, 1069

Lucas, R., \& Liszt, H. S. 2002, A\&A, 384, 1054

Maier, J. P., Lakin, N. M., Walker, G. A. H., \& Bohlender, D. A. 2001, ApJ, 553, 267

McCall, B. J., Hinkle, K. H., \& Geballe, T. R. et al. 2002, ApJ, 567,391

Meyer, D. M., \& Roth, K. C. 1991, ApJ, 376, L49

Millar, T., Farquhar, P., \& Willacy, K. 1997, A\&AS, 121, 139

Morton, D. C. 1975, ApJ, 197, 85

Nguyen, T. K., Hartquist, T. W., \& Williams, D. A. 2001, A\&A, 366, 662

Pirronello, V., Liu, C., Roser, J. E., \& Vidali, G. 1999, A\&A, 344,681

Roueff, E., Felenbok, P., Black, J. H., \& Gry, C. 2002, A\&A, 384,629

Savage, B. D., Drake, J. F., Budich, W., \& Bohlin, R. 1977, ApJ, 216

Savage, B. D., \& Sembach, K. R. 1996, Ann. Rev. A\&A, 34

Smith, A. M., Bruhweiler, F. C., Lambert, D., et al. 1991, ApJ, $377,61 \mathrm{~L}$

Snow, T. P. 1977, ApJ, 216, 724

Snow, T. P., Lamers, H. J. G. L. M., \& Joseph, C. L. 1987, ApJ, 321, 952

Snow, T. P., \& Witt, A. N. 1996, ApJ, 468, L65

Turner, B. E. 1995a, ApJ, 444, 708

Turner, B. E. 1995b, ApJ, 455, 556

Turner, B. E. 1996a, ApJ, 461, 246

Turner, B. E. 1996b, ApJ, 468, 694

Turner, B. E. 1998, ApJ, 501, 731

Turner, B. E. 2000, ApJ, 542, 837

Turner, B. E., Pirogov, L., \& Minh, Y. C. 1997, ApJ, 483, 235

Turner, B. E., Terzieva, R., \& Herbst, E. 1999, ApJ, 518, 699

van Dishoeck, E. F. 1998, in The molecular astrophysics of stars and galaxies, ed. T. W. Hartquist, \& D. Williams, 53

van Dishoeck, E. F., \& Black, J. H. 1986, ApJS, 62, 109

van Dishoeck, E. F., Phillips, T. G., Black, J. H., \& Gredel, R. 1991, ApJ, 366, 141

Vidali, G., Roser, J., \& Pirronello, V. 1999, in $\mathrm{H}_{2}$ in Space, ed. F. Combes, \& G. Pineau des Forêts (Cambridge University Press), E14

Viti, S., Williams, D. A., \& O'neill, P. T. 2000, A\&A, 354, 1062

Wagenblast, R. 1992, MNRAS, 259, 155

Wagenblast, R., \& Williams, D. A. 1993, in Dust and Chemistry in Astronomy, ed. T. J. Millar, \& D. A. Williams (Institute of Physics Publishing Ltd.)

Wagenblast, R., \& Williams, D. A. 1996, Astrophys. \& Sp. Sci., 236, 257

Wagenblast, R., Williams, D. A., Millar, T. J., \& Nejad, L. A. M. 1993, MNRAS, 260, 420

Weingartner, J. C., \& Draine, B. T. 1999, ApJ, 517, 292

Williams, D. A. 1998, in Faraday Discussion 109 (Royal Society of Chemistry), 1

Williams, D. A., Clary, D. C., Farebrother, A., et al. 1999, in $\mathrm{H}_{2}$ in Space, ed. F. Combes, \& G. Pineau des Forêts (Cambridge University Press) 